\title{
HIP for AM - Optimized Material Properties by HIP
}

\author{
Magnus Ahlfors $^{1, a}{ }^{*}$, Fouzi Bahbou ${ }^{2, b}$, Anders Eklund ${ }^{3, c}$, Ulf Ackelid ${ }^{2, d}$ \\ ${ }^{1}$ Quintus Technologies LLC, Lewis center, OH, USA \\ ${ }^{2}$ Arcam AB, Mölndal, Sweden \\ ${ }^{3}$ Quintus Technologies AB, Västerås, Sweden \\ amagnus.ahlfors@quintusteam.com, ${ }^{b}$ fouzi.bahbou@arcam.com, \\ canders.eklund@quintusteam.com, dulf.ackelid@freemelt.com
}

Keywords: Hot Isostatic Pressing (HIP), Additive Manufacturing, Titanium

\begin{abstract}
An investigation of HIP parameters for EBM Ti-6Al-4V has been performed by Arcam $\mathrm{AB}$ and Quintus Technologies $\mathrm{AB}$ with the aim to maximize the strength of the HIP:ed material. A lower HIP temperature of $800^{\circ} \mathrm{C}$ and a higher pressure of $200 \mathrm{MPa}$ gives the highest strength and is also enough to eliminate all internal defects. By printing material with intentionally induced porosity combined with an optimized HIP cycle the highest strength can be obtained.

\section{Introduction}

Hot isostatic pressing (HIP) is widely used today to eliminate internal defects in metallic materials produced by powder bed fusion. The internal defects are mostly lack-of-fusion defects generated during the printing process and entrapped gas porosity coming from the powder particles. These defects act like stress concentrations and crack initiation points in the material, which decreases the material properties. By eliminating these defects within the material, the ductility and especially the fatigue properties are improved [1-5]. Figure 1 shows a cross section of an EBM Ti-6Al-4V material before and after HIP where the typical effect of HIP:ing in terms of defect elimination can be seen. In Figure 2, typical fatigue data of as printed and HIP:ed material of EBM Ti-6Al-4V is shown and it is evident that the HIP process gives much improved fatigue properties compared to as-printed material. This data is generated by Arcam.
\end{abstract}
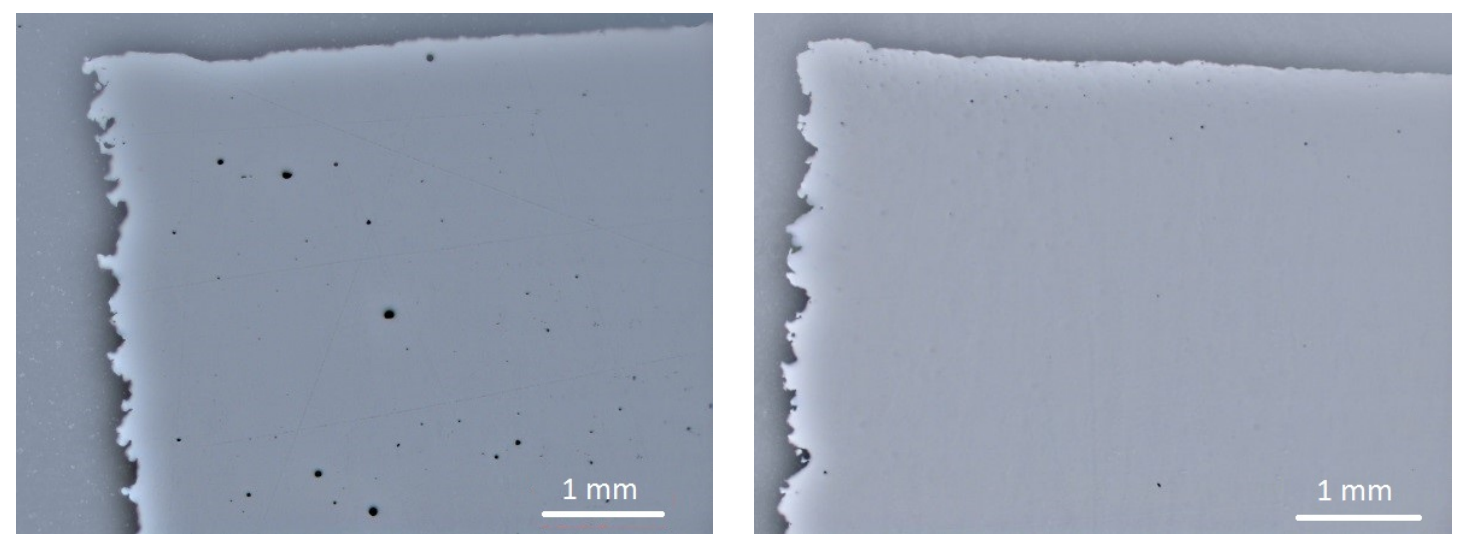

Figure 1 - Micrographs of EBM Ti-6Al-4V before HIP to the left and after HIP to the right 


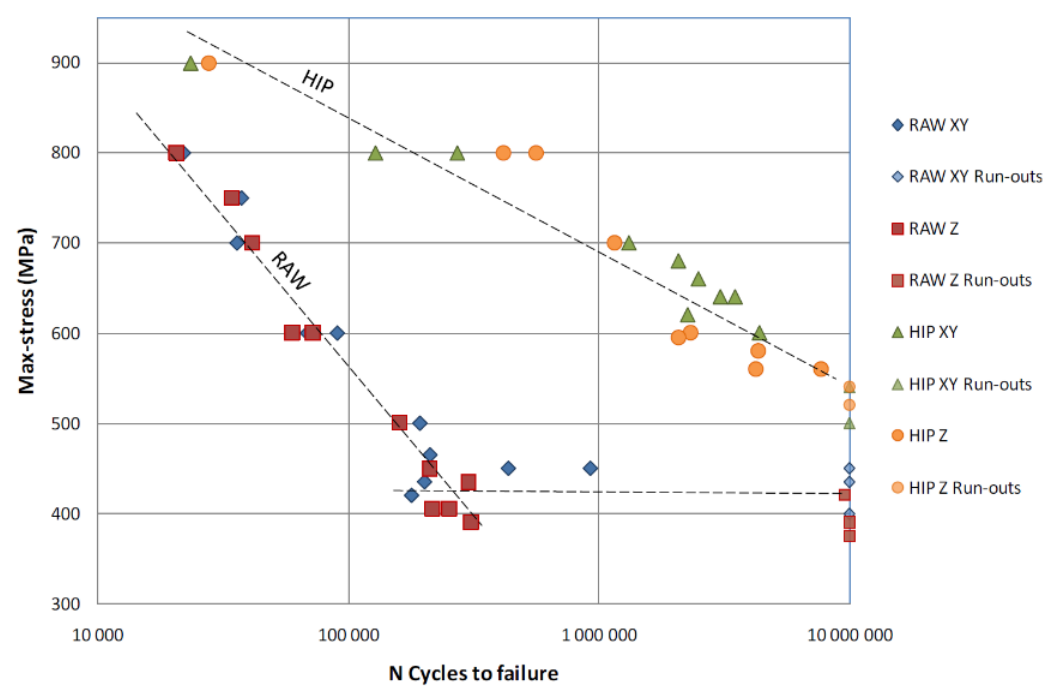

Figure 2 - Fatigue data for EBM Ti-6Al-4V, courtesy of Arcam

The solidification rates in the EBM process are in the order of $10^{3}-10^{5} \mathrm{~K} / \mathrm{s}$ which is very high [6]. The extremely fast solidification generates a very fine microstructure which gives the material a high strength. Any conventional heat treatment at an elevated temperature for a significantly long time, like HIP:ing, will coarsen the microstructure due thermodynamic driving forces. This coarsening of the microstructure will decrease the strength of the material, which is not preferable. The development within EBM printing equipment over the last years has made the as-printed microstructures even finer, which makes this challenge even more significant for the modern EBM machines. In Figure $3 \mathrm{a}$ ) and b) the microstructure of as printed material compared to HIP:ed EBM Ti-6Al-4V is shown. The coarsening of the microstructure after HIP is evident. Figure $3 \mathrm{a}$ ) and c) shows the difference between the microstructures produced by an older Arcam s12 machine compared to a newer Arcam Q10 machine.
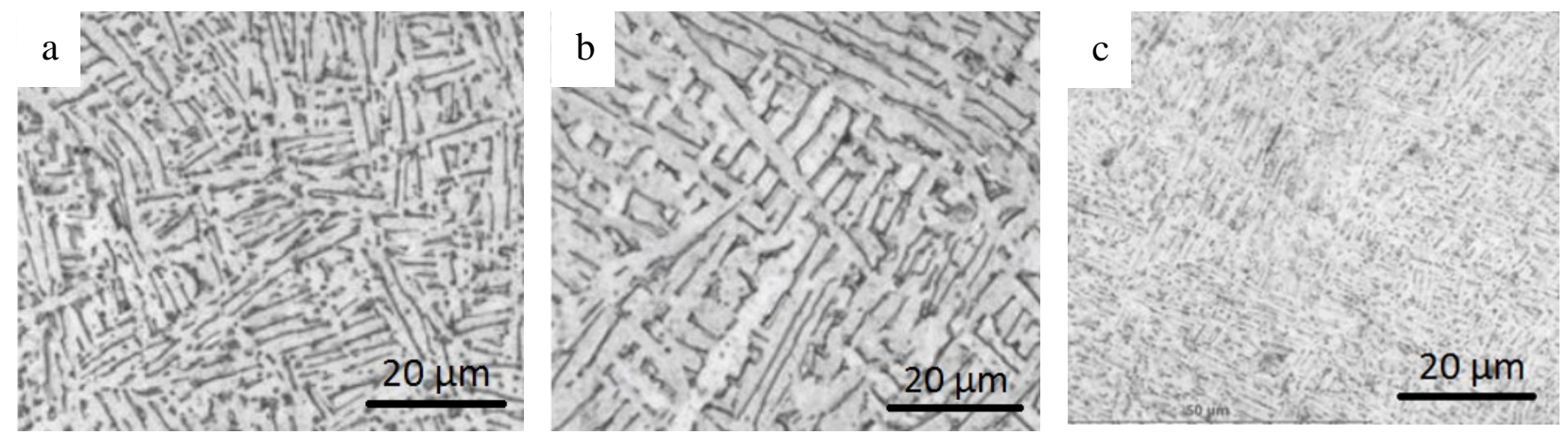

Figure 3 - Microstructures of EBM Ti-6Al-4V a) As-printed with Arcam s12 b) After HIP $\left(920^{\circ} \mathrm{C}, 1000 \mathrm{bar}, 2 \mathrm{~h}\right)$ with Arcam s12 c) As-printed with Arcam Q10

For Ti-6Al-4V produced by selective laser melting (SLM), the same coarsening of the microstructure and thus decrease of strength has been seen. As reported by Leuders [1] the tensile strength of SLM Ti-6Al-4V is decreased by any kind of elevated temperature process including HIP:ing as shown in Figure 4. 


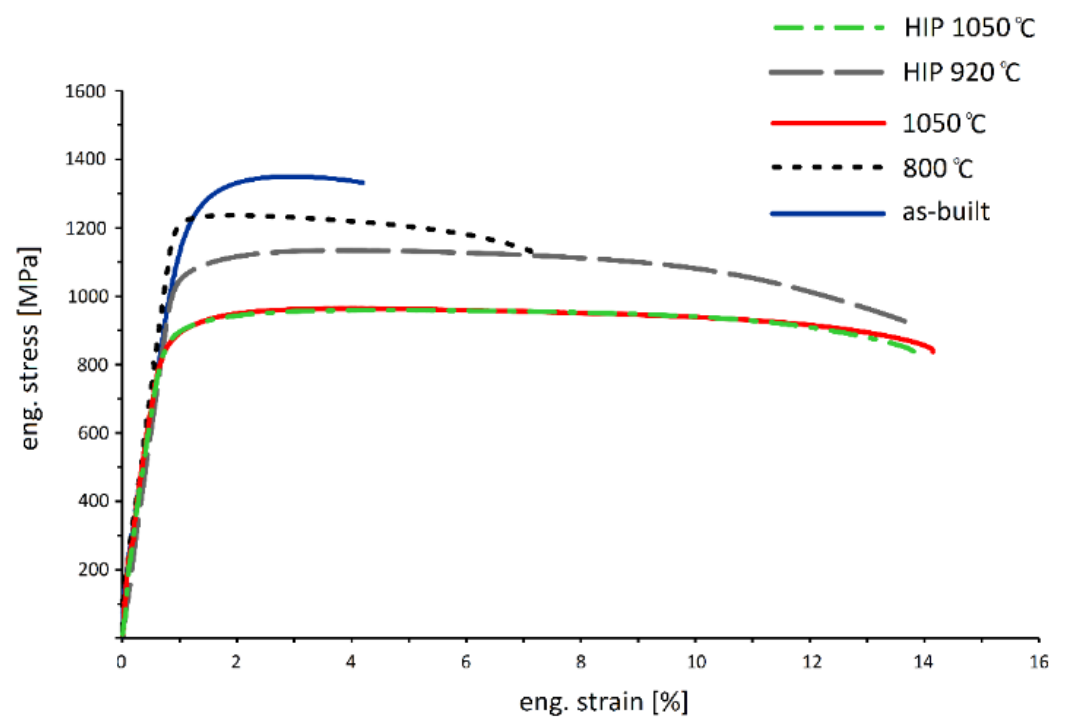

Figure 4 - Tensile test data of SLM Ti-6Al-4V in different conditions [1]

The ASTM standard for additive manufactured Ti-6Al-4V by powder bed fusion, F2924-14, specifies a HIP temperature between 895 and $955^{\circ} \mathrm{C}$, a minimum pressure of $100 \mathrm{MPa}$ and a minimum hold time of 2 hours. The widely used HIP parameters for AM Ti-6Al-4V is $920^{\circ} \mathrm{C}$, $100 \mathrm{MPa}$ and 2 hours hold time, thus within the standard specification. However, these HIP parameters were developed for cast Ti-6Al-4V before today's commercial AM processes existed and it is not obvious that these HIP parameters are optimal for AM material.

With this background, a study of HIP parameters for EBM Ti-6Al-4V was made by Arcam and Quintus Technologies with the purpose to evaluate if other HIP parameters could be used to eliminate all defects but have a lower influence on the fine as-printed microstructure. The approach of this study is to evaluate lower HIP temperatures.

\section{Experimental}

The HIP parameter study consisted of 6 different HIP treatments according to Table 1 together with as-printed material as reference. The test specimens for the study were $15 \mathrm{~mm}$ diameter rods printed with standard EBM printing parameters and powder and 10 test specimens for each condition were used for the evaluation.

Table 1 - The 6 different HIP treatments used in the study

\begin{tabular}{|l|l|l|l|l|}
\hline Variant & $\begin{array}{l}\text { Temperature } \\
{\left[{ }^{\circ} \mathrm{C}\right]}\end{array}$ & $\begin{array}{l}\text { Pressure } \\
{[\mathrm{MPa}]}\end{array}$ & Hold time [hours] & $\begin{array}{l}\text { Cooling } \\
{[\mathrm{K} / \mathrm{min}]}\end{array}$ \\
\hline 1 & 920 & 100 & 2 & $\sim 30$ \\
\hline 2 & 920 & 100 & 2 & $\sim 1500$ \\
\hline 3 & 880 & 100 & 2 & $\sim 30$ \\
\hline 4 & 840 & 100 & 2 & $\sim 30$ \\
\hline 5 & 800 & 100 & 2 & $\sim 30$ \\
\hline 6 & 800 & 200 & 2 & $\sim 30$ \\
\hline
\end{tabular}


The results of the different HIP variants were evaluated by density, internal defect analysis and tensile data in xy and z-direction. The density was measured by helium pycnometry and with Archimedes principle, here called water intrusion. The internal defects in the material were evaluated by optical microscopy and the tensile data was attained with standard tensile testing.

As an extension of the HIP parameter study, material printed with intentional internal porosity was also HIP:ed and analyzed. The intentional induced defects were generated by printing with a larger line off-set up to $0.4 \mathrm{~mm}$ instead of the standard $0.2 \mathrm{~mm}$. In Figure 5 the macrostructure of material printed with the standard $0.2 \mathrm{~mm}$ line off-set and $0.4 \mathrm{~mm}$ line off-set is shown and a large difference in number of defects can be observed.
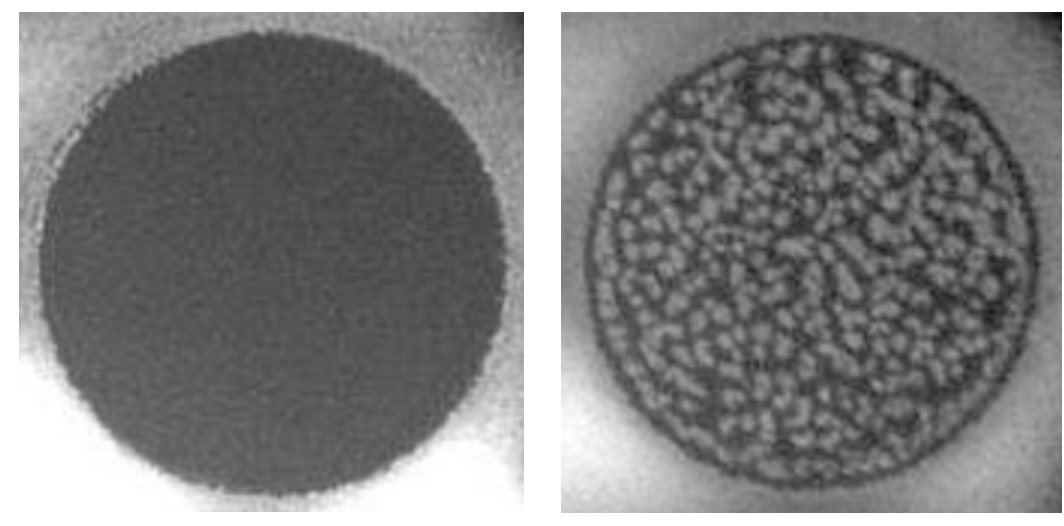

Figure 5 - Defects analyzed with LayerQam. To the left with $0.2 \mathrm{~mm}$ line off-set and $0.4 \mathrm{~mm}$ to the right.

\section{Results}

In Figure 6 the results from the density measurements are presented. As can be seen, all HIP variants gives $\sim 100 \%$ density. The material printed with standard parameters also shows $\sim 100$ $\%$ density in the as-printed state. There is still porosity in the material but a relatively small amount as shown in Figure 1 so that no influence is visible in the density measurements. For the material printed with larger line off-set the density is significantly lower than the standard printed material with up to $8 \%$ porosity for the material printed with $0.4 \mathrm{~mm}$ line off-set. However, these samples are also fully densified to $100 \%$ density with HIP despite the low starting density. The contour of the porous printed specimens was built with standard printing parameters to achieve a gas tight surface for the specimens. A gas tight outer surface of the part to be HIP:ed is required to ensure full densification by the HIP process without any encapsulation of the part. 


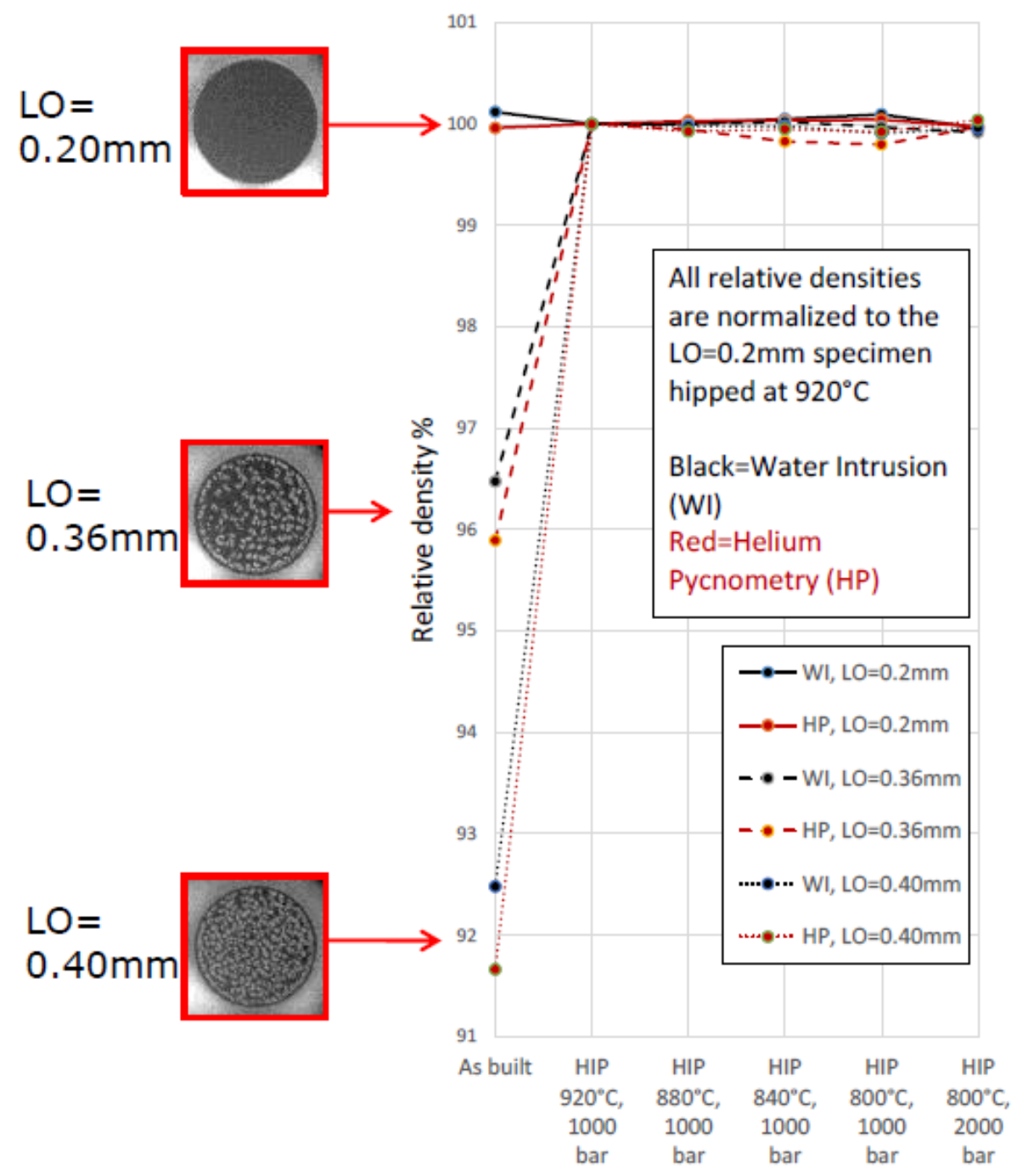

Figure 6-Density measurements results

The results for the evaluation of defects with optical microscopy showed that HIP variants 1 , 2, 3 and 6 gave full defect elimination. HIP variants number 4 and 5 with 840 and $800{ }^{\circ} \mathrm{C}$ and $100 \mathrm{MPa}$ showed some small remaining defects which were not eliminated by HIP. The density measurements in Figure 6 also tend to show the same result.

The tensile test results for the material printed with standard parameters are shown in Figure 7. The results show that there is a significant increase in strength when going towards lower HIP temperatures. The HIP cycles at $800{ }^{\circ} \mathrm{C}$ gives the best strength and the ductility is not influenced significantly by the different HIP variants. 


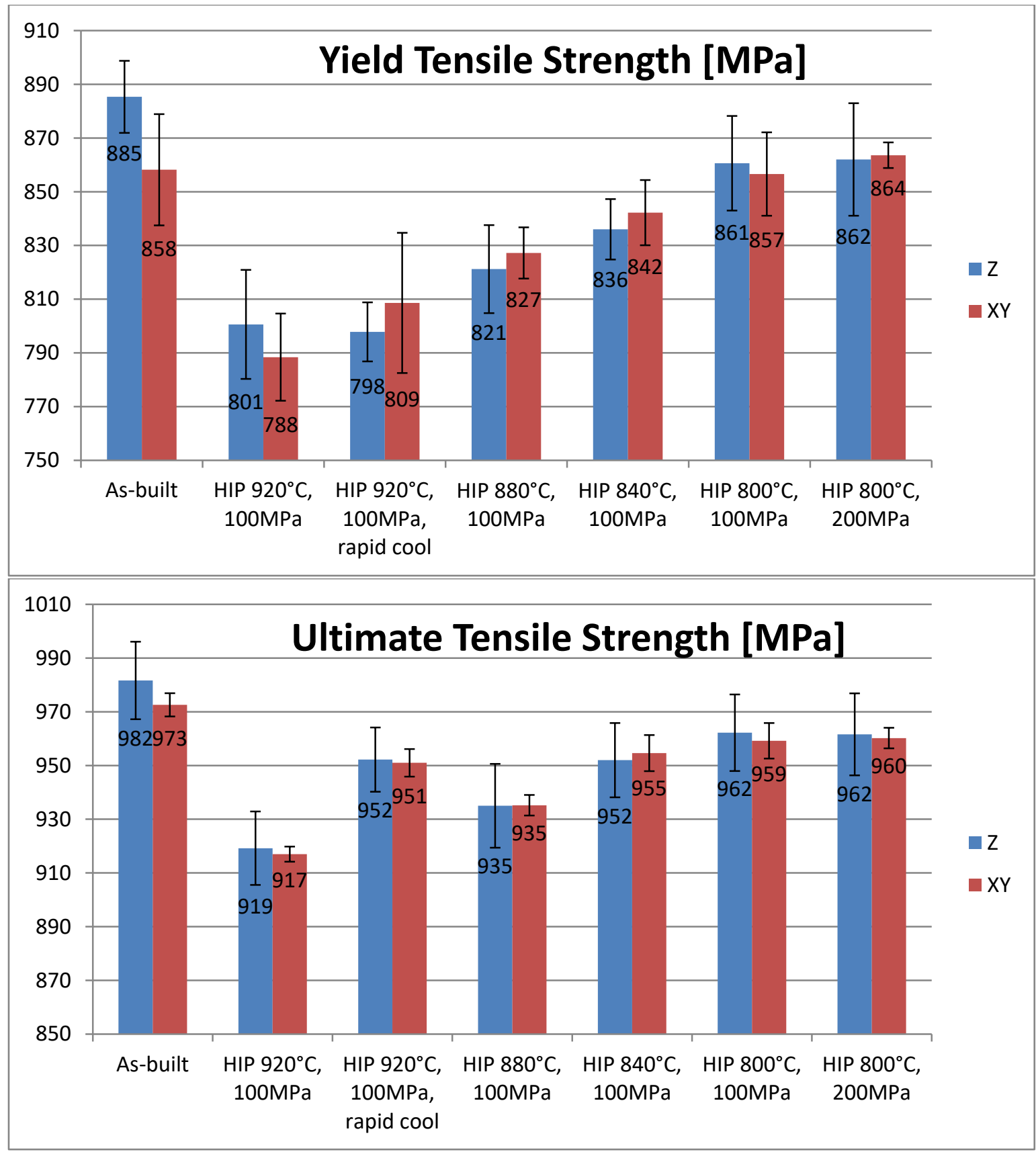




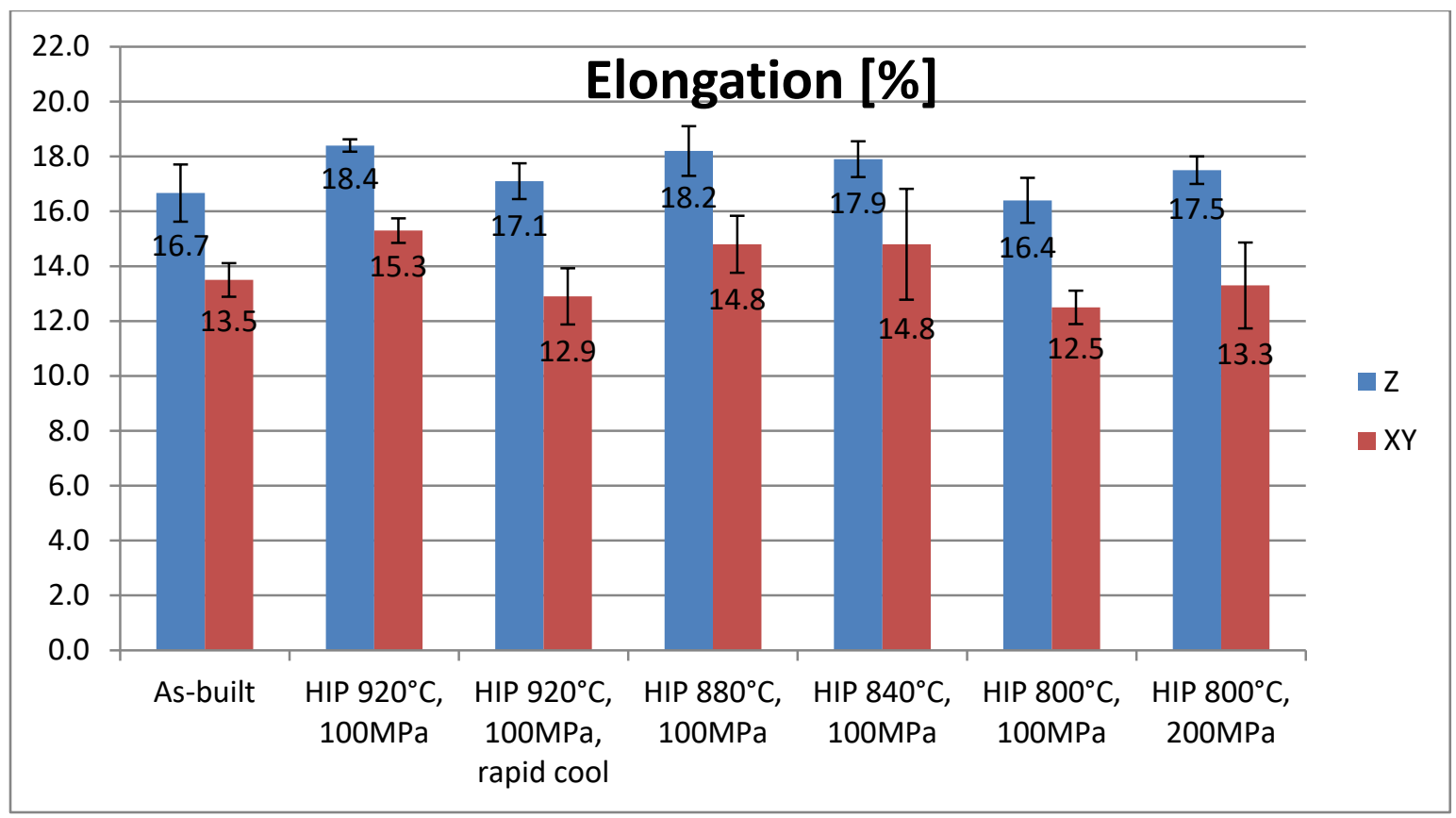

Figure 7 - Tensile data for standard printed material

The tensile test results for the material printed with intentional defects are shown in Figure 8 together with the data from the standard printed material. The first interesting observation that can be made is that the strength of the porous printed material is higher than the standard printed material even though it contains $\sim 8 \%$ porosity. However, the ductility of the porous printed material is significantly reduced by the $\sim 8 \%$ porosity. After HIP, the strength values are reduced but always higher for the porous printed material compared to the standard printed material. For the porous printed material HIP:ed with optimized parameters at $800{ }^{\circ} \mathrm{C}$ the strength is higher than the as-printed material printed with standard parameters. In addition, the very low ductility in the as-printed porous material is fully recovered by the HIP process making a material with higher strength than the as-printed material and the same ductility. 


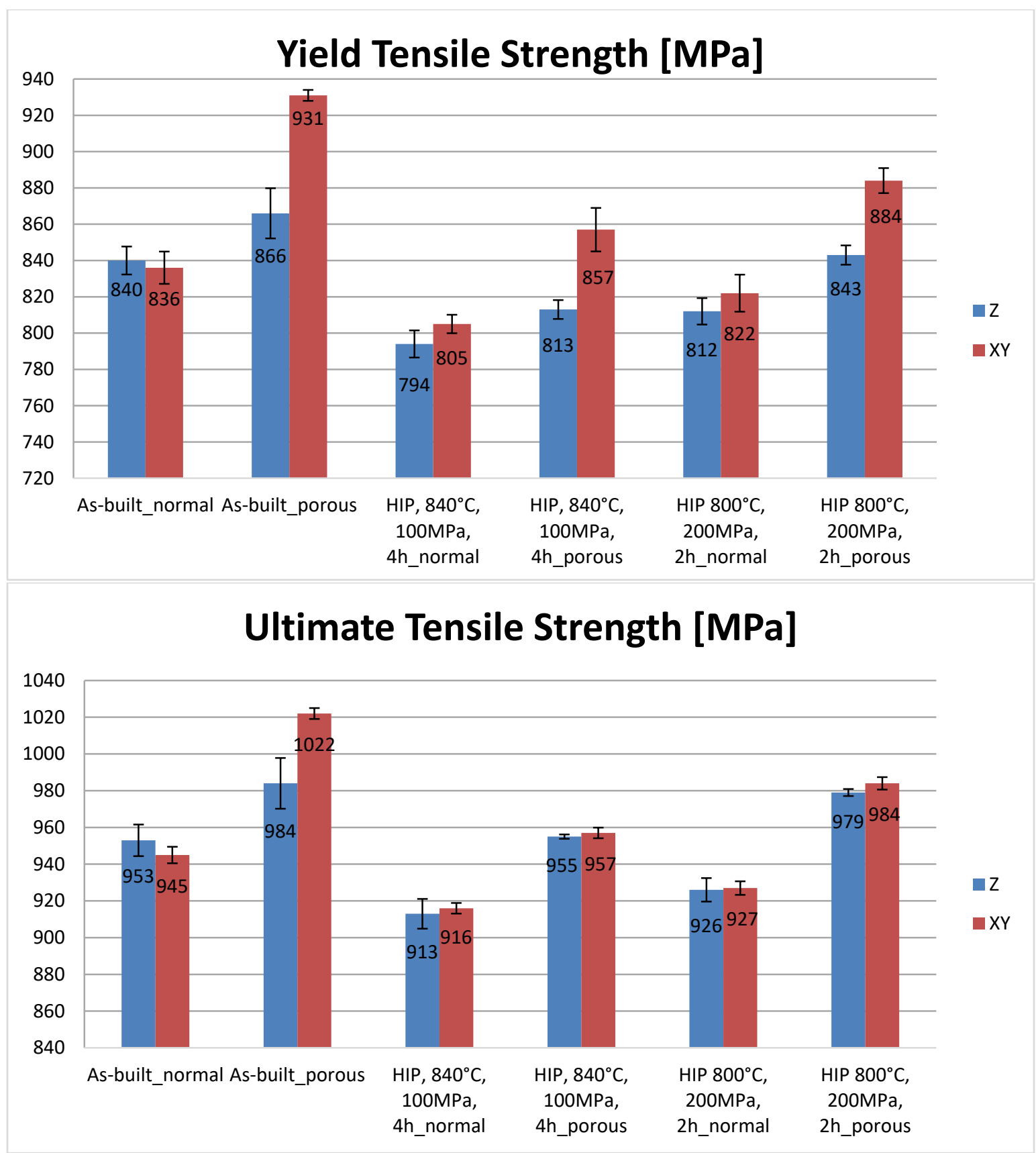




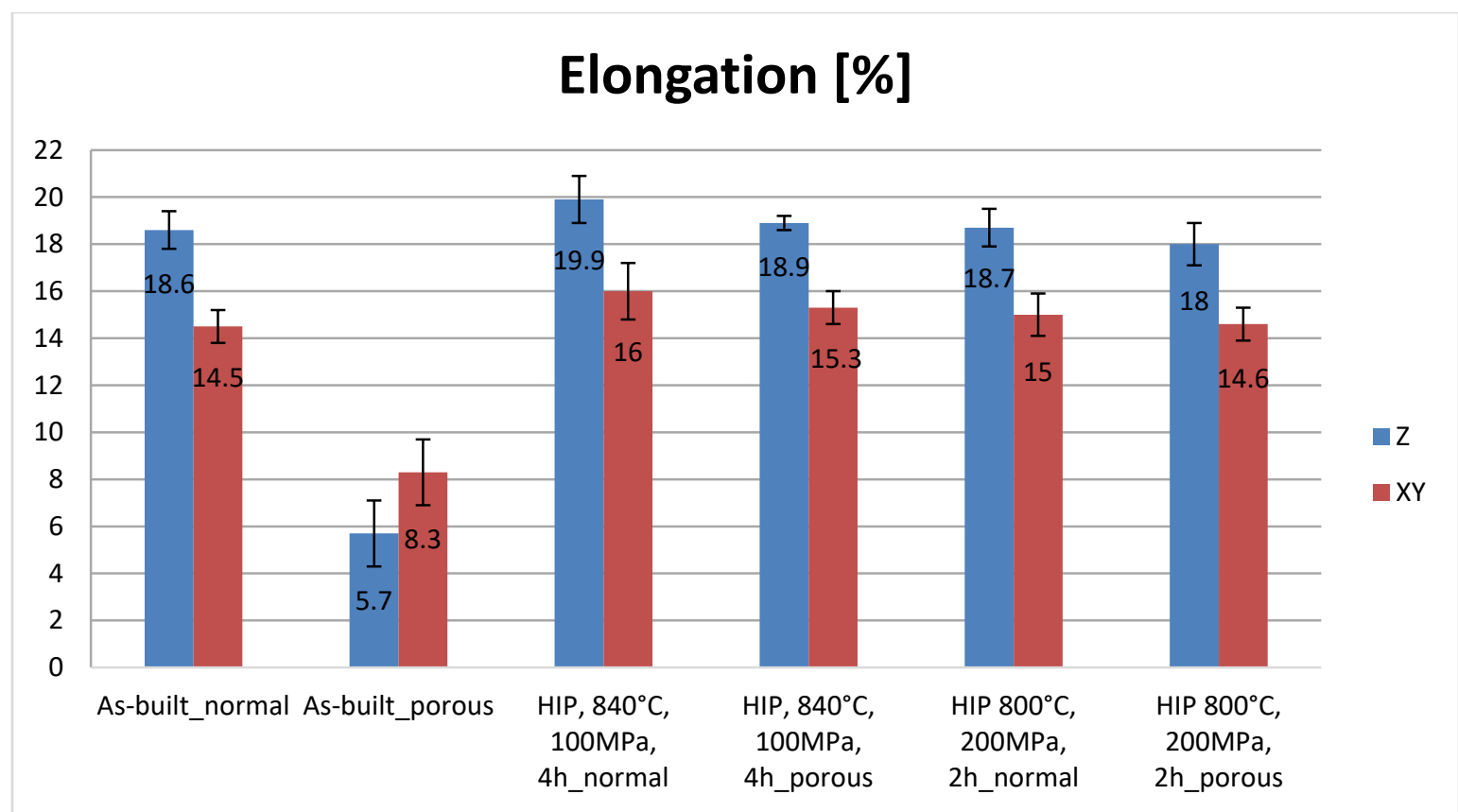

Figure 8 - Tensile data for standard and porous printed material

An interesting question is, why the material printed with $~ 8 \%$ porosity shows higher tensile strength than a virtually fully dense material printed with the standard parameters? An explanation for that could be the increased line off-set of $0.4 \mathrm{~mm}$ which will generate a smaller melt pool in the printing process and so the layers are less re-melted. This reduced re-melting of the material results in an even finer as-printed microstructure, which gives a higher strength. The smaller melt pool and less re-melting also reduces the loss of aluminum due to evaporation during the printing process which, which is positive in terms of strength.

\section{Conclusions}

The conclusion from this study is that it is possible to HIP EBM manufactured Ti-6Al-4V with a HIP temperature as low as $800{ }^{\circ} \mathrm{C}$ and still get full density in the material if the HIP pressure is increased from 100 to $200 \mathrm{MPa}$ with an unchanged hold time of 2 hours. These HIP parameters will give a significantly higher strength compared to the standard HIP parameters of $920{ }^{\circ} \mathrm{C}, 100$ $\mathrm{MPa}$ and 2 hours due to reduced coarsening of the fine microstructure.

It can also be concluded that printing with a larger line-offset induces more porosity in the material during the printing process but also gives higher strength due to less energy input into the material. The result is that optimized HIP parameters for AM in combination optimized printing parameters for HIP will lead to a material which is stronger than the as-printed standard material but shows same ductility level. It can be assumed that the HIP:ed material will also have the already well-known significant increase in fatigue properties compared to the as-printed material.

The final conclusion from this study is that HIP, heat treatments and other processes should be investigated for AM material in order to achieve optimal properties, and these post treatments cannot just be copied from e. g. the casting industry because additive manufacturing is something different than the conventional manufacturing processes. Another important point is if HIP:ing is used, the printing process can be adjusted for HIP to achieve optimum material properties. In 
case of a subsequent HIP process, there is no need to achieve $99.9 \%$ theoretical density in the as-printed part since the densification result after HIP:ing will be the same even with $92 \%$ asprinted density.

\section{References}

[1] S. Leuders, On the mechanical performance of structures manufactured by Selective Laser Melting: Damage initiation and propagation, University of Paderborn, Germany, as presented at AMPM2014, MPIF, USA

[2] N. Hrabe et al., Fatigue properties of a titanium alloy (Ti-6Al-4V) fabricated via electron beam melting (EBM): Effects of internal defects and residual stress, International Journal of Fatigue Volume 94, Jan 2017. https://doi.org/10.1016/j.ijfatigue.2016.04.022

[3] A. Kirchner et al., Mechanical Properties of Ti-6Al-4V Additively Manufactured by Electron Beam Melting, EuroPM2015, Reims, France

[4] J. J Lewandowski M. Seifi, Metal Additive Manufacturing: A Review of Mechanical Properties, Annual Review of Materials Research, July 2016

[5] J. Haan et al, Effect of Subsequent Hot Isostatic Pressing on Mechanical Properties of ASTM F75 Alloy Produced by Selective Laser Melting, EuroPM2014, Salzburg, Austria

[6] S. Al-Bermani, M. Blackmore, W. Zhang, I. Todd, The origin of microstructural diversity, texture, and mechanical properties in electron beam melted Ti-6Al-4V, Metallurgical and Materials Transactions A 41 (2010) 3422-3434. https://doi.org/10.1007/s11661-010-0397-x 